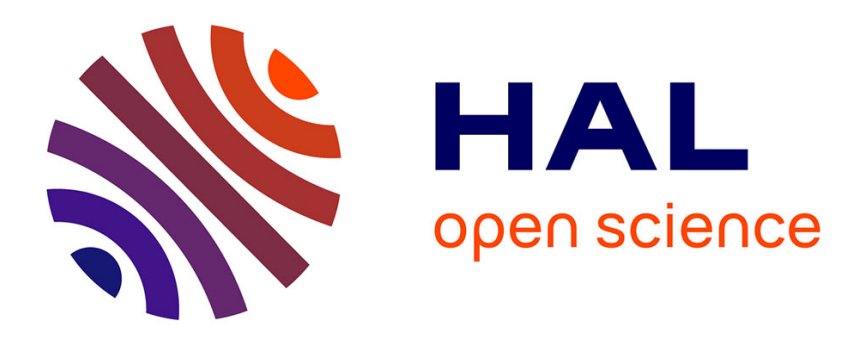

\title{
Swelling of the various parts of a polymer chain in a good solvent
}

Bertrand Duplantier

\section{To cite this version:}

Bertrand Duplantier. Swelling of the various parts of a polymer chain in a good solvent. Journal de Physique Lettres, 1985, 46 (16), pp.751-758. 10.1051/jphyslet:019850046016075100 . jpa-00232894

\section{HAL Id: jpa-00232894 https://hal.science/jpa-00232894}

Submitted on 1 Jan 1985

HAL is a multi-disciplinary open access archive for the deposit and dissemination of scientific research documents, whether they are published or not. The documents may come from teaching and research institutions in France or abroad, or from public or private research centers.
L'archive ouverte pluridisciplinaire HAL, est destinée au dépôt et à la diffusion de documents scientifiques de niveau recherche, publiés ou non, émanant des établissements d'enseignement et de recherche français ou étrangers, des laboratoires publics ou privés. 
Classification

Physics Abstracts

$05.20-05.40-61.40 \mathrm{~K}-64.70$

\title{
Swelling of the various parts of a polymer chain in a good solvent
}

\author{
B. Duplantier
}

Service de Physique Théorique, CEN-Saclay, 91191 Gif-sur-Yvette Cedex, France

(Reçu le 13 mai 1985, accepté le 26 juin 1985)

\begin{abstract}
Résumé. - Nous considérons une chaîne polymère isolée en bon solvant et nous calculons le gonflement d'une partie quelconque de celle-ci. Nous montrons que ce gonflement est beaucoup plus important dans la partie centrale de la chaîne, et qu'il décroît abruptement lorsque l'on atteint l'une de ses extrémités. Nous donnons les expressions des moyennes de toutes les puissances de la distance entre deux points quelconques de la chaîne, au premier ordre en $\varepsilon=4-d$, où $d$ est la dimension de l'espace. Des indices critiques en sont déduits.
\end{abstract}

\begin{abstract}
We consider an isolated polymer chain in a good solvent and calculate the swelling of an arbitrary part of it. We show that this swelling is much more important in the central part of the chain, and decreases abruptly when one reaches one of its extremities. We give the expressions of the averages of all the powers of the distance between two arbitrary points of the chain, at first order in $\varepsilon=4-d$, $d$ being the space dimension. Critical indices are deduced from it.
\end{abstract}

\section{Introduction.}

Our aim is to study the swelling of a given part of a polymer chain immersed in a good solvent. This swelling is by no means uniform along the chain, in contradistinction with the case of a Brownian chain. For the latter, the average distance between two arbitrary points of the chain depends only on the difference of abscissa of the two points, and not of the position along the Brownian chain. The problem we consider has not been treated up to now in a satisfactory way. Yamakawa [1] made calculations at first order in the excluded volume parameter $z$ but drew erroneous conclusions from it, claiming for a decrease of the swelling near the centre of the chain. A popular model of uniform swelling was also proposed by Peterlin [2], Benoit [3] and Ptitsyn [4]. It has been recently criticized [5] (see also [6]), concerning the form factor of the chain. Here we find it interesting to clarify this issue by using a direct approach.

We work with the continuous model $[7,8]$. The chain is represented by a curve $\mathbf{r}(s)$ in $d$-dimensional space. The parameter $s$, proportional to the number of links, is in fact a "Brownian area ". One has $0 \leqslant s \leqslant S$, where $S$ is the total Brownian area of the chain. We shall make here a systematic and simple calculation of the averages $\left\langle\left\|\mathbf{r}\left(s^{\prime}\right)-\mathbf{r}\left(s^{\prime \prime}\right)\right\|^{2 n}\right\rangle$ of the $2 n$th powers of the dis- 
tance between two points of abscissae $s^{\prime}$ and $s^{\prime \prime}$ along the chain. We shall measure these quantities with respect to the size $X$ of the polymer chain, defined by :

$$
\left\langle\|\mathbf{r}(S)-\mathbf{r}(0)\|^{2}\right\rangle=\mathrm{d} X^{2},
$$

or with respect to higher order powers of the end-to-end distance.

If we denote by $t=\frac{\left|s^{\prime}-s^{\prime \prime}\right|}{S}, 0 \leqslant t \leqslant 1$, the fraction of the chain we consider, we expect a result of the scaling form :

$$
\left\langle\left\|\mathbf{r}\left(s^{\prime}\right)-\mathbf{r}\left(s^{\prime \prime}\right)\right\|^{2 n}\right\rangle=t^{2 v n}\left\langle\|\mathbf{r}(S)-\mathbf{r}(0)\|^{2 n}\right\rangle\left[1+g^{*} \mathbb{F}_{n}^{\cdot}+\cdots\right]
$$

where $v$ is the usual critical index. Here $t^{2 v n}\left\langle\|\mathbf{r}(S)-\mathbf{r}(0)\|^{2 n}\right\rangle$ is exactly the swollen size the fraction $t$ would have if it were free to swell and not embedded in a larger chain. $g^{*}$ is the dimensionless second virial coefficient of the chain, which for a very long Kuhnian chain has the value $g^{*}=\varepsilon / 8+\cdots$ where $\varepsilon=4-d$. Thus $g^{*} \mathbb{F}_{n}^{*}$ really appears as an excess swelling due to the insertion of the fraction $t$ inside the large Kuhnian chain. As we shall see, $\mathbb{F}_{n}^{*}$ depends crucially on the position of the fraction along the chain. $n$ is a priori an integer but we shall perform the analytical continuation to any real $n, n>-2$.

Incidentally, we note that some results concerning the probability distribution of the internal distances already exist, for the critical indices [9] and for the form of the distribution [10]. We shall discuss them later.

\section{Calculation.}

We evaluate the generating function :

$$
h[\mathbf{q}]=\left\langle\mathrm{e}^{i \mathbf{q} \cdot\left[\mathbf{r}\left(\mathbf{s}^{\prime}\right)-\mathbf{r}\left(s^{\prime \prime}\right)\right]}\right\rangle
$$

in the framework of the continuous model. It can be written :

$$
h[\mathbf{q}]=\frac{3\left(\mathbf{q}, s^{\prime}, s^{\prime \prime}\right)}{3\left(\mathbf{0}, s^{\prime}, s^{\prime \prime}\right)}
$$

where $3\left(\mathbf{q}, s^{\prime}, s^{\prime \prime}\right)$ denotes the partition function of a continuous polymer chain with an insertion of wave vector $\mathbf{q}$ at the points $s^{\prime}$ and $s^{\prime \prime}$. A similar quantity has been considered in a recent publication [5]. The diagrams contributing to (3) to first order in the interaction parameter $b$ are given in figure 1. One finds then :

$$
h[\mathbf{q}]=\mathrm{e}^{-y t}-z\left[I_{1}(y, t)+I_{2}\left(y, t, x^{\prime}\right)+I_{2}\left(y, t, x^{\prime \prime}\right)+I_{3}\left(y, t, x^{\prime}, x^{\prime \prime}\right)\right]
$$

with the variables

$$
\begin{gathered}
y=q^{2} S / 2, \quad z=(2 \pi)^{-d / 2} b S^{2-d / 2}, \quad t=\frac{s^{\prime}-s^{\prime \prime}}{S}\left(s^{\prime}>s^{\prime \prime}\right), \\
x^{\prime}=\frac{S-s^{\prime}}{S}, \quad x^{\prime \prime}=\frac{s^{\prime \prime}}{S} \\
t+x^{\prime}+x^{\prime \prime} \equiv 1 .
\end{gathered}
$$

The functions

$$
I_{1}(y, t)=\int_{0}^{t} \mathrm{~d} t^{\prime} t^{\prime-d / 2}\left(t-t^{\prime}\right)\left[\mathrm{e}^{y t^{\prime}}-1\right] \mathrm{e}^{-y t}
$$




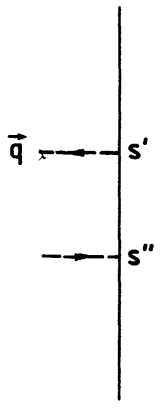

0

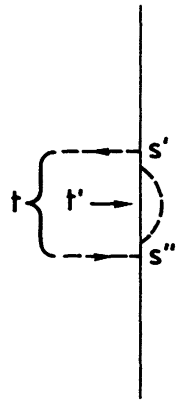

1
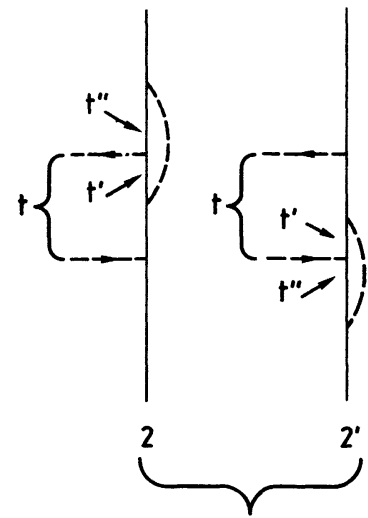

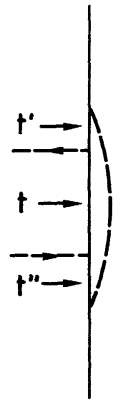

3

Fig. 1. - The diagrams contributing to $h[\mathbf{q}]$ at first order.

$$
\begin{aligned}
& I_{2}\left(y, t, x^{\prime}\right)=\int_{0}^{t} \mathrm{~d} t^{\prime} \int_{0}^{x^{\prime}} \mathrm{d} t^{\prime \prime}\left(t^{\prime}+t^{\prime \prime}\right)^{-\mathrm{d} / 2}\left[\mathrm{e}^{y \frac{t^{\prime 2}}{t^{\prime}+t^{\prime \prime}}}-1\right] \mathrm{e}^{-y t} \\
& I_{3}\left(y, t, x^{\prime}, x^{\prime \prime}\right)=\int_{0}^{x^{\prime}} \mathrm{d} t^{\prime} \int_{0}^{x^{\prime \prime}} \mathrm{d} t^{\prime \prime}\left(t+t^{\prime}+t^{\prime \prime}\right)^{-\mathrm{d} / 2}\left[\mathrm{e}^{y \frac{t^{2}}{t+t^{\prime}+t^{\prime \prime}}}-1\right] \mathrm{e}^{-y t}
\end{aligned}
$$

correspond respectively to the diagrams $1,2,3$ of figure 1 . Of these expressions, $I_{1}$ is the only one to diverge when $\varepsilon=4-d \rightarrow 0$. Thus the expression (5) for $h[\mathbf{q}]$ has to be renormalized. This is done by expressing it as a function of the physical quantity [5] :

$$
x=q^{2} X^{2} / 2
$$

instead of $y=q^{2} S / 2 . X$ is the size (1) of the swollen chain. One has :

$$
x / y=X^{2} / S=\mathfrak{x}_{0}(z)
$$

where the swelling factor $\mathfrak{X}_{0}(z)$ reads [8] :

$$
\begin{aligned}
& x_{0}(z)=1+a_{d} z+\cdots \\
& a_{d}=\int_{0}^{1} \mathrm{~d} t^{\prime}\left(1-t^{\prime}\right) t^{\prime-d / 2}=\frac{2}{\varepsilon}-1+\cdots
\end{aligned}
$$

for $d=4-\varepsilon$.

Eliminating $y$ in favour of $x$ (Eq. (8)) in equation (5) we find, to first order in $z$, the renormalized quantity :

$$
h[\mathbf{q}]=\mathrm{e}^{-t x}-z\left[I_{1}(x, t)-a_{d} t x \mathrm{e}^{-t x}+I_{2}\left(x, t, x^{\prime}\right)+I_{2}\left(x, t, x^{\prime \prime}\right)+I_{3}\left(x, t, x^{\prime}, x^{\prime \prime}\right)\right] .
$$

Now this expression is finite when $\varepsilon=4-d$ goes to zero. In this way, using previous results $[5,8]$, it is possible to write :

$$
z=g+\cdots
$$

where $g$ is the second virial coefficient (in scaling form) [8]. Moreover for $z \rightarrow \infty$, we know that :

$$
g \rightarrow g^{*}=\frac{\varepsilon}{8}+\cdots
$$


We now expand the well-defined quantity (10) in powers of $t x$, taking the limit $d \rightarrow 4$ for the various functions which appear in equations (7), (9) and (10). We find, after some algebra, the series expansion :

$$
\begin{aligned}
h[\mathbf{q}]= & +\sum_{n \geqslant 1} \frac{(-x t)^{n}}{n !}\left\{1-g\left[-n \ln t+\left(1-\delta_{n l}\right) \sum_{2 \leqslant n^{\prime} \leqslant n}(-1)^{n^{\prime}} C_{n}^{n^{\prime}} \frac{1}{n^{\prime}} \frac{1}{n^{\prime}-1}+\right.\right. \\
& +\sum_{1 \leqslant n^{\prime} \leqslant n}(-1)^{n^{\prime}} C_{n}^{n^{\prime}} \frac{1}{n^{\prime}+1}\left[\frac{1}{n^{\prime}}-J_{n^{\prime}}\left(a^{\prime}\right)\right]+\sum_{1 \leqslant n^{\prime} \leqslant n}(-1)^{n^{\prime}} C_{n}^{n^{\prime}} \frac{1}{n^{\prime}+1}\left[\frac{1}{n^{\prime}}-J_{n^{\prime}}\left(a^{\prime \prime}\right)\right]+ \\
& \left.\left.+\sum_{1 \leqslant n^{\prime} \leqslant n}(-1)^{n^{\prime}} C_{n}^{n^{\prime}} \frac{1}{n^{\prime}+1} \frac{1}{n^{\prime}}\left[\left(1+a^{\prime}+a^{\prime \prime}\right)^{-n^{\prime}}-\left(1+a^{\prime}\right)^{-n^{\prime}}-\left(1+a^{\prime \prime}\right)^{-n^{\prime}}+1\right]\right]\right\}
\end{aligned}
$$

with

$$
J_{n}(a)=\int_{0}^{1} \mathrm{~d} t^{\prime} \frac{t^{\prime 2 n}}{\left(t^{\prime}+a\right)^{n+1}}
$$

and

$$
a^{\prime}=x^{\prime} / t, \quad a^{\prime \prime}=x^{\prime \prime} / t, \quad 1+a^{\prime}+a^{\prime \prime}=t^{-1} .
$$

Using definition (8) in this expansion (12) gives the expansion of $h[\mathbf{q}]$ in powers of $q^{2}$. By definition (3), this expansion generates the averaged powers (2). We find :

$$
\left\langle\left\|\mathbf{r}\left(s^{\prime}\right)-\mathbf{r}\left(s^{\prime \prime}\right)\right\|^{2 n}\right\rangle=\frac{\Gamma(n+d / 2)}{\Gamma(d / 2)} 2^{n}\left(t^{1+g+\cdots}\right)^{n} X^{2 n}\left[1+g \mathbb{F}_{n}\left(a^{\prime}, a^{\prime \prime}\right)\right]
$$

where the function $\mathbb{F}_{n}$ reads

$$
\begin{aligned}
& \mathbb{F}_{n}\left(a^{\prime}, a^{\prime \prime}\right)=-S_{n}-3 S_{n}-F_{n}\left(a^{\prime}\right)-F_{n}\left(a^{\prime \prime}\right)-G_{n}\left(a^{\prime}, a^{\prime \prime}\right) \\
& S_{n}=(n+1) \sum_{1 \leqslant n^{\prime} \leqslant n} \frac{1}{n^{\prime}}-2 n \\
& S_{n}=-\sum_{1 \leqslant n^{\prime} \leqslant n} \frac{1}{n^{\prime}}-\frac{1}{n+1}+1 \\
& F_{n}(a)=-\sum_{1 \leqslant n^{\prime} \leqslant n}(-1)^{n^{\prime}} \mathrm{C}_{n}^{n^{\prime}} \frac{1}{n^{\prime}+1} J_{n^{\prime}}(a) \\
& G_{n}\left(a^{\prime}, a^{\prime \prime}\right)=\sum_{1 \leqslant n^{\prime} \leqslant n}(-1)^{n^{\prime}} \mathrm{C}_{n}^{n^{\prime}} \frac{1}{n^{\prime}+1} \frac{1}{n^{\prime}}\left[\left(1+a^{\prime}+a^{\prime \prime}\right)^{-n^{\prime}}-\left(1+a^{\prime}\right)^{-n^{\prime}}-\left(1+a^{\prime \prime}\right)^{-n^{\prime}}\right] .
\end{aligned}
$$

The result in (14) and (15) contains all the information we need. However, if we want to set it in the form (2) we may normalize it not by $X^{2 n}$ but by the average $\left\langle\|\mathbf{r}(S)-\mathbf{r}(0)\|^{2 n}\right\rangle$. The latter can be obtained directly from equations (14) and (15) by setting $t=1, a^{\prime}=a^{\prime \prime}=0$. One finds that $\mathbb{F}_{n}(0,0)=-S_{n}$, and therefore :

$$
\left\langle\|\mathbf{r}(S)-\mathbf{r}(0)\|^{2 n}\right\rangle=\frac{\Gamma(n+d / 2)}{\Gamma(d / 2)} 2^{n} X^{2 n}\left(1-g S_{n}\right) .
$$


Thus we may rewrite (14) in the simpler scaling form :

$$
\left\langle\left\|\mathbf{r}\left(s^{\prime}\right)-\mathbf{r}\left(s^{\prime \prime}\right)\right\|^{2 n}\right\rangle=\left(t^{1+g+\cdots}\right)^{n}\left\langle\|\mathbf{r}(S)-\mathbf{r}(0)\|^{2 n}\right\rangle\left[1+g \mathbb{F}_{n}^{*}\left(a^{\prime}, a^{\prime \prime}\right)\right]
$$

where $\mathbb{F}_{n}^{*}\left(a^{\prime}, a^{\prime \prime}\right)$ is the « excess swelling function » expected in (2) :

$$
\mathbb{F}_{n}^{\cdot}\left(a^{\prime}, a^{\prime \prime}\right)=-3 S_{n}^{\cdot}-F_{n}\left(a^{\prime}\right)-F_{n}\left(a^{\prime \prime}\right)-G_{n}\left(a^{\prime}, a^{\prime \prime}\right) .
$$

Incidentally, we note that for $n=1, \mathbb{F}_{n=1}$ and $\mathbb{F}_{n=1}^{\cdot}$ coincide. The formula (17) has just the form (2) since, at this order, $2 v=1+\varepsilon / 8+\cdots=1+g^{*}+\cdots$

\section{Particular results.}

Two simple cases can be immediately obtained from (14) and (15).

3. $1 n=1$.

All the sums in (15) reduce to a single term and the swelling function $\mathbb{F}_{n=1}$ reads :

$$
\mathbb{F}_{n=1}\left(a^{\prime}, a^{\prime \prime}\right)=a^{\prime} \ln \left(1+1 / a^{\prime}\right)+a^{\prime \prime} \ln \left(1+1 / a^{\prime \prime}\right)+\frac{1}{2} t-\frac{1}{2}
$$

where $a^{\prime}, a^{\prime \prime}$, defined in (13), take their value on the $\left[0, t^{-1}-1\right]$ interval, for a given $t$. Similar expressions appear in different contexts [11].

In order to compare different values of $t$, it is convenient to introduce the variable :

$$
u=t \frac{a^{\prime}}{1-t}=\frac{s^{\prime}}{(1-t) S}, \quad u \in[0,1]
$$

which measures the displacement of one extremity of the chain fraction with respect to the available displacement along the chain. With this variable, (14) or (17) can be rewritten in the form :

with

$$
\left\langle\left\|\mathbf{r}\left(s^{\prime}\right)-\mathbf{r}\left(s^{\prime \prime}\right)\right\|^{2}\right\rangle=t^{1+\varepsilon / 8+\cdots} \mathrm{d} X^{2}\left[1+\frac{\varepsilon}{8} \mathbb{F}_{n=1}[t, u]+\cdots\right]
$$

$$
\mathbb{F}_{n=1}[t, u]=\frac{1-t}{t}\left\{u \ln \left[1+\frac{t}{(1-t) u}\right]+(1-u) \ln \left[1+\frac{t}{(1-t)(1-u)}\right]-\frac{1}{2}(1-t)\right\}
$$

The curves giving $\mathbb{E}_{n=1}[t, u]$ as a function of $u$ for different values of $t$ are given in figures 2,3. One clearly sees the drastic increase of swelling when moving towards the centre of the chain. One notices also this remarquable fact : any finite part of the chain is more swollen than the entire chain. This is natural : a fraction of the chain feels the external repulsive segments which add to its swelling, when compared to an entire chain of the same Brownian size. In a similar way, a fraction near the centre of the chain is swollen by more external segments than the same fraction located near the extremities of the chain.

The limit $t \rightarrow 0$ corresponds to an infinitesimally small fraction of a finite chain, or conversely to a finite fraction of an infinite chain.

$$
\mathbb{F}_{n=1}[t \rightarrow 0, u]
$$




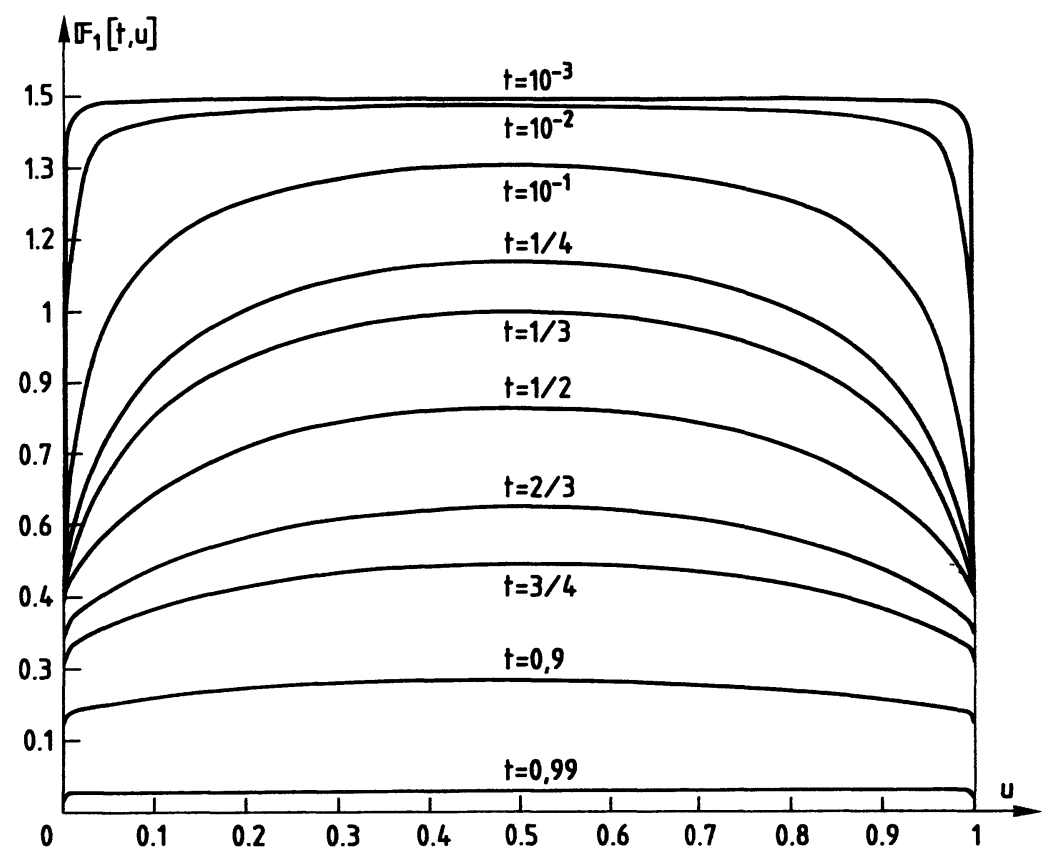

Fig. 2. - The excess swelling functions $\mathbb{F}_{n=1}[t, u]$ versus the positions $u=\frac{s^{\prime}}{(1-t) S}, 0 \leqslant u \leqslant 1$, of the fraction $t$ along the chain. For $t=0$, the function is discontinuous, taking the values $1 / 2$ or $3 / 2$.

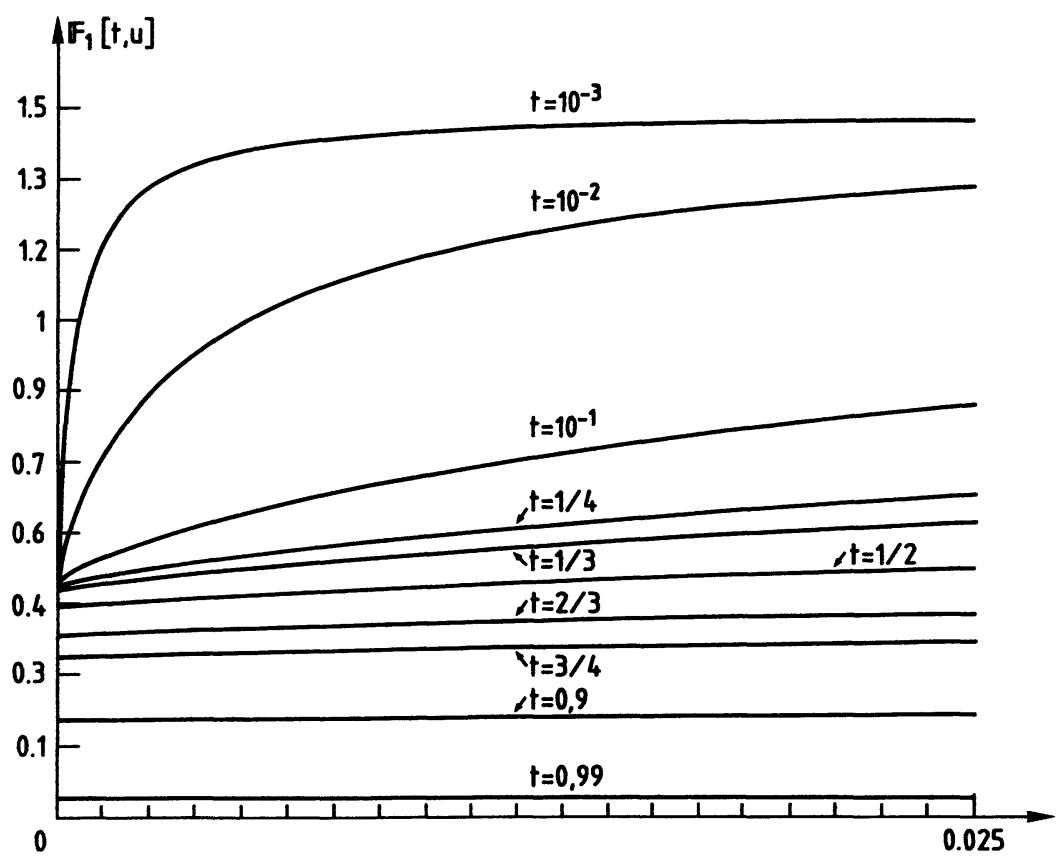

Fig. 3. - The same functions $\mathbb{F}_{n=1}[t, u]$ at a broader scale, for $0 \leqslant u \leqslant 0.025$, and for the same set of values of $t$. 
is then a discontinuous function :

$$
\mathbb{F}_{n=1}[t \rightarrow 0, u]=\left\{\begin{array}{lll}
1 / 2 & \text { if } & u=0 \\
3 / 2 & \text { if } & u \neq 0
\end{array} .\right.
$$

This intriguing behaviour in the limit $t \rightarrow 0$ can be easily and intuitively understood. One explores an infinitesimal fraction of the chain, with respect to which the two remaining arms are infinite, whatever the position of the fraction is. So the excess swelling function is constant along the chain. However, if the fraction is exactly at the extremity of the chain, there is an inversion of limits and the fraction, however small, feels the absence of repulsive chain on one of its sides. Naturally, for a finite part of the chain, which would be the case in an experiment, all these effects are softened, but they are still significant as shown in figures 2,3 . This shows clearly the difference of swelling between the very extremity of the chain and the interior of the chain. For $t=1$, one recovers the whole chain, and one has identically $\mathbb{F}_{n=1}[t=1, u]=0$. This was expected since $\mathbb{F}_{n=1}$ precisely measures the swelling in excess with respect to the global swelling of the chain.

\section{2 arbitrary.}

Returning to equations (14) and (15), we shall focus here on three particular geometrical situations :

a) $t=1$ : the whole chain. One has then necessarily $a^{\prime}=a^{\prime \prime}=0$, and as already seen above :

$$
\mathbb{F}_{n}(0,0)=-S_{n}
$$

b) $t \rightarrow 0, a^{\prime}=0, a^{\prime \prime} \rightarrow \infty$, which corresponds to the extremity of an infinite chain. Then, from equation (15) one gets :

$$
\mathbb{F}_{n}(0, \infty)=-S_{n}-S_{n}
$$

c) $t \rightarrow 0, a^{\prime}=a^{\prime \prime} \rightarrow \infty$, which corresponds to an internal fraction of an infinite chain. Then one has :

$$
\mathbb{F}_{n}(\infty, \infty)=-S_{n}-3 S_{n}^{*}
$$

Now, the sums $S_{n}$ and $S_{n}^{*}$ appearing in (15) can be represented by :

$$
\begin{aligned}
& S_{n}=(n+1)[\psi(n+1)+C]-2 n \\
& S_{n}^{*}=1-\psi(n+1)-C-\frac{1}{n+1},
\end{aligned}
$$

where $\psi(z)=\frac{\mathrm{d}}{\mathrm{d} z} \ln \Gamma(z)$, and where $C$ is Euler's constant. Thus the analytical continuation of $S_{n}$ and $S_{n}$ to non integer values $z$ of $n$ is straight-forward. We can thus write directly the general result for the three cases $a, b, c$ :

$$
\left\langle\left\|\mathbf{r}\left(s^{\prime}\right)-\mathbf{r}\left(s^{\prime \prime}\right)\right\|_{a}^{2 z}\right\rangle=\frac{\Gamma(z+d / 2)}{\Gamma(d / 2)} 2^{z}\left(t^{\nu} X\right)^{2 z}\left[1+g \mathbb{F}_{z}(a)\right]
$$

for $z>-2$, with

$$
\mathbb{F}_{z}(a)=-(z+1)[\psi(z+1)+C]+2 z+a\left[\psi(z+1)+C+\frac{1}{z+1}-1\right]
$$

where the index $a$ takes the three values $a=0,1,3$ for cases $a, b, c$ respectively. 
The quantity (25) is actually still defined at the first singularity of $\psi(z+1)$ which occurs for $z=-1$. One finds then the interesting finite result :

$$
\left\langle\left\|\mathbf{r}\left(s^{\prime}\right)-\mathbf{r}\left(s^{\prime \prime}\right)\right\|_{a}^{-2}\right\rangle=\left(t^{\nu} X\right)^{-2} \frac{1}{d-2}[1-g(1+a)] .
$$

Thus equation (24) holds for any real $z>-2$.

Let us end by indicating some results concerning the probability distribution $P_{a}(x), x=r / t^{v} X$, for the distance $r$ between the two extremities of a chain fraction $t$. At short distances, one expects $P_{a}(x) \sim x^{\theta_{a}}$ where the $\theta_{a}$ 's are contact critical indices [9], corresponding to the three different physical situations $\mathrm{a}, \mathrm{b}, \mathrm{c}$. These indices are naturally related to the first singularity at $z=-2$ of the moments (24), (25). From its study we obtain the set of critical indices :

$$
\begin{aligned}
& \theta_{a}=\frac{1+a}{4} \varepsilon+\cdots \\
& a=0,1,3
\end{aligned}
$$

in agreement with reference [9].

On the contrary, at large distances, one expects $P_{a}(x)$ to behave like $P_{a}(x) \sim x^{\sigma_{a}} \exp \left[-A x^{\frac{1}{1-v}}\right]$, where $A$ is some constant and where $\sigma_{a}$ is another set of critical indices. For $a=0$, this index was calculated in [12]. These indices are naturally related to the behaviour of the moments (24), (25) for large values of $z$. From its study, we obtain the set of indices :

$$
\sigma_{a}=\frac{1+a}{4} \varepsilon+\cdots
$$

For $a=0,1,3$.

They coincide with the short range indices (27) but this holds true only at first order in $\varepsilon$.

The method we presented here is the simplest one for getting the set of averages (14) and studying the swelling.

We have also directly calculated the probability distribution and thus checked the preliminary results of reference [10]. We shall present these calculations elsewhere.

\section{References}

[1] Yamakawa, H., Modern Theory of Polymer Solutions (Harper and Row) 1971, p. 93.

[2] Peterlin, A. J., J. Chem. Phys. 23 (1955) 2464.

[3] Benort, H., C. R. Hebd. Séan. Acad. Sci. Paris 245 (1957) 2244.

[4] Ptrtsyn, O. B., Zh. Fiz. Khim 31 (1957) 1091.

[5] Des Cloizeaux, J., Duplantier, B., J. Physique Lett. 46 (1985) L-457.

[6] Witten Jr, T. A., J. Chem. Phys. 76 (1982) 3300.

Ohta, T., OONo, Y., Freed, K., Phys. Rev. A 25 (1982) 2801.

[7] Edwards, S. F., Proc. Phys. Soc. 85 (1965) 613.

[8] des Cloizeaux, J., J. Physique 42 (1981) 635.

[9] Des Cloizeaux, J., J. Physique 41 (1980) 223.

[10] Oono, Y., OHTa, T., Phys. Lett. A 85 (1981) 480.

[11] MiYAKe, A., FreED, K., Macromolecules 16 (1983) 1228 ;

Oono, Y., "Statistical Physics of polymer solutions " preprint (University of Illinois at Urbana).

[12] Des CloizeauX, Phys. Rev. A 10 (1974) 1665. 